\title{
Leaders
}

\section{Cloning of local growth factors involved in the determination of muscle mass}

Unlike the cells of many tissues, muscle and neuronal cells do not replicate throughout life. Therefore there has to be an effective mechanism of inducing local repair and preventing cell death. We have identified and cloned two growth factors that are expressed by muscle when it is subjected to activity which are derived from the insulin like growth factor-I (IGF-I) gene by alternative splicing. ${ }^{12}$ One isoform, muscle L.IGF-I, is very similar to the liver type of IGF-I. The other is a new growth factor that could only be detected in exercised or stretched muscle. This has been called mechano growth factor (MGF) to distinguish it from liver IGF-I which has a systemic mode of action. The structure of the cDNA of this isoform indicates that it has different exons from the liver types and is not glycosylated. ${ }^{1}$ Therefore it is smaller and has a shorter half life than liver IGF-I unless it is bound to its tissue binding protein. Unlike hormones, the site and mode of action of growth factors are determined to a large extent by their specific binding proteins.

Both muscle isoforms are upregulated by stretch and overload and the evidence indicates that, during exercise, muscle L.IGF-I contributes significantly to circulating levels of IGF-I. ${ }^{23} \mathrm{MGF}$, however, appears to be designed for local action and does not enter the blood stream in any quantity. It has a 52 base insert in the $\mathrm{E}$ domain which alters the reading frame of the $3^{\prime}$ end, which results in it binding to a different binding protein which exists in the interstitial tissue spaces of muscle and neuronal tissue. This would be expected to localise its action as it would be unstable in the unbound form; this is important as its production would not unduly perturb blood sugar levels.

Although MGF production in response to stretch during exercise is apparently to induce local repair, its overexpression in situations that involve pronounced overload-for example, weight lifting - results in hypertrophy. Therefore it has an adaptative as well as a protective function. The mechanism whereby cells respond to mechanical signals must involve a mechanochemical transduction mechanism to link the physical signal with the activation or repression of certain genes. Recently, there have been a number of studies on other cell types that implicate the cytoskeleton in mechanochemical transduction. These have been reviewed by Ingebar. ${ }^{4}$ Experiments have shown that in dystrophic muscle, including that in $\mathrm{mdx}$ mice (a model for dystrophin deficient dystrophies) and dydy mice (model for autosomal dystrophies), MGF is not expressed as it is in normal mice muscle when stretched. ${ }^{5}{ }^{6}$ In both types of dystrophy, the cytoskeletal dystrophin complex is defective and so apparently is the mechanotransduction system. At the C-terminus, dystrophin is attached to an elaborate array of different proteins, which are in turn attached to the extracellular matrix through laminin (merosin) one of which is missing in the autosomal dystrophies. Also associated with this complex are neuronal nitric oxide synthase and a tyrosine kinase, therefore it seems inconceivable that this elaborate structure is present merely to stiffen the membrane. The defects in the rather specialised cytoskeleton in muscle apparently results in inadequate production of local IGF-I, and the failure to maintain muscle mass is a possible cause for all the dystrophies.

Using an isolated heart preparation, we were able to detect the production of the MGF peptide using a specific antibody just two hours after the heart had been subjected to a pressure overload. ${ }^{7}$ This growth factor appears to be involved in protecting heart as well as skeletal muscle by inducing local repair and preventing apoptosis. There is also evidence that it is also involved in maintaining nervous tissue, ${ }^{8}$ as IGF-I is known to be transported within neurones. Therefore the possibility exists that motor neurone maintenance is facilitated by IGF-I produced by the active muscles that they innervate. ${ }^{9}$ Using a specific antibody that we have generated, we found the MGF splice variant in the central nervous system, which is another tissue in which there is no cell replacement and in which continuous local repair is very important. In addition, the systemic type of IGF-I produced by active muscles (L.IGF-I) and its beneficial effect on other types of tissue, as well as the protective and adaptative effects of the autocrine variant (MGF), provides a basis for understanding the beneficial effects of exercise on general health. ${ }^{10}$

Recently, we placed MGF cDNA in an engineered gene and injected it into muscles of the laboratory mouse. Somewhat to our surprise, we found a $20 \%$ increase in muscle mass in two weeks. ${ }^{11}$ At the same time, a group in Philadelphia introduced the liver type IGF-I into muscles using a similar approach and they also reported a $20 \%$ increase but only after four months. Interestingly, this group reported greater increases within four months in the muscles of older mice as compared with the normal aging controls, indicating that the age related loss of muscle mass (sarcopenia) is associated with decreased IGF-I levels. As systemic levels of growth hormone and liver IGF-I decrease with age, this can be supplemented by the potent MGF version produced by active muscles. This emphasises the need to remain active during later years.

Clearly MGF is very potent and much more effective in increasing muscle mass than the liver type of IGF-I. This may be good news for the elderly suffering from advanced sarcopenia and for children suffering from muscular dystrophy and other musculoskeletal problems, but it will be open to abuse. If one intramuscular injection results in a $20 \%$ increase in that muscle within a short time, it may be predicted that it will be misused-for example, for resculpturing the body for athletic performance or other non-medical purposes. As MGF and muscle L.IGF-I are produced naturally by the body during activity, it would be difficult to detect whether an individual performance had been enhanced by gene therapy or achieved by hard training. At present, there is apparently considerable misuse of recombinant liver type IGF-I, and it is naive to think that this would not be extended to include the more potent form. It is to be hoped that the method we have in mind for testing for this form of abuse (which cannot be disclosed at this juncture) will be effective in detecting exogenous MGF.

This research was supported by a grant from the Wellcome Trust. 
GEOFFREY GOLDSPINK

Department of Anatomy and Developmental Biology, Division of Biomedical Sciences, The Royal Free and University College Medical School, University of London, Rowland Hill Street, London NW3 2PF United Kingdom

email:chaplin@rfhsm.ac.uk

1 Yang SY, Alnaqeeb $\mathrm{M}$, Simpson $\mathrm{H}$, et al. Cloning and characterisation of an IGF-1 isoform expressed in skeletal muscle subjected to stretch. 7 Muscle Res Cell Motil 1996;17:487-95.

2 McKoy G, Ashley W, Mander J, et al. Expression of IGF-1 splice variants and structural genes in rabbit skeletal muscle are induced by stretch and stimulation. $\mathcal{F}$ Physiol (Lond) 1999;516:583-92.

3 Brahm H, Piehl-Aulin K, Saltin B, et al. Net fluxes over working thigh of hormones, growth factors and biomarkers of bone metabolism during lasting dynamic exercise. Calcif Tissue Int 1997;60:175-80.

4 Ingebar D. Tensegrity: architectural basis of cellular mechanotransduction. Annu Rev Physiol 1997;59:575-99.
5 Goldspink G, Yang SY, Skarli M, et al. Local growth regulation is associated with an isoform of IGF-1 that is expressed in normal rabbit, mouse and human muscles but not in dystrophic mouse muscles when subjected to stretch. F Physiol (Lond) 1996;162:496P

6 Yang SY, Alnaqeeb M, Simpson H, et al. Changes in muscle fibre type, muscle mass and IGF- 1 gene expression in rabbit skeletal muscle subjected to stretch. F Anat 1997;190:613-22.

7 Skarli M, Yang SY, Bouloux P, et al. Upregulation and alternative splicing of the IGF-1 gene in the rabbit heart following a brief pressure/volume overload. F Physiol (Lond) 1998;192:509P.

8 D'Ercole AJ, Ye P, Calikoglu AS, et al. The role of the insulin-like growth factors in the central nervous system. Mol Neurobiol 1996;13:227-55.

9 von Bartheid CS, Byers MR, Williams R. Autograde transport and axodendritic transfer in the developing visual system. Nature 1996;379:832-3.

10 Goldspink G. Molecular mechanisms involved in the determination of muscle fibre mass and phenotype. Advances in Exercise and Sports Physiology 1999;5:27-39.

11 Barton-Davies ER, Shoturma DL, Musaro A, et al. Viral mediated expression of insulin-like growth factot I blocks the aging-related loss of skeletal muscle function. Proc Natl Acad Sci USA 1998;95:15603-7.

\section{Exercise for cancer patients: a new challenge in sports medicine}

In the past, physicians usually advised patients with chronic diseases to rest and avoid physical effort. These recommendations were empirical: as most chronic diseases are associated with functional changes resulting in an impairment of physical performance, exercise in this group of patients may generate fatigue, breathlessness, and tachycardia. Therefore, avoiding physical activity results in less discomfort.

However, in the last few years, scientific evidence has dramatically changed our ideas about exercise for patients with chronic diseases. In the late 1960s, the inclusion of physical activity in rehabilitation programmes for patients who had had myocardial infarction set a milestone and opened up new perspectives for the use of exercise in treatment for chronic diseases. Now, it is a well established fact that excessive rest and lack of physical activity may result in severe deconditioning and thus reduce the functional status and quality of life of the chronically ill. Furthermore, numerous studies have shown that exercise is an effective means for counteracting several of the negative effects that chronic diseases have on physical performance. As a result of this evidence, exercise is actually considered an essential component in the treatment of several illnesses including peripheral vascular disorders, chronic obstructive lung disease, ischaemic myocardial disease, and heart failure. Moreover, the value of exercise for rehabilitation has been widely recognised; indeed, many institutions offer exercise programmes for transplantation recipients and for patients after myocardial infarction or with chronic renal failure. However, until recently, less was known about the feasibility and effects of exercise programmes for cancer patients during and after treatment.

The role of exercise in oncological rehabilitation programmes has thus far been mostly limited to physical treatment addressing specific impairments caused-for example, by amputation or surgery. However, the medical attitude regarding exercise for cancer patients is changing fast. The recent world class performances of athletes who have been treated for cancer have focussed attention on the effects of training on the physical performance of cancer patients. Moreover, recent studies have shown that physical activity may improve both the quality of life and mood and the physical performance of cancer patients during and after treatment.

Regular physical activity has been shown to increase the performance status in breast cancer patients treated with conventional chemotherapy ${ }^{1}$ and in patients after bone marrow transplantation. ${ }^{2}$ It has also been shown to reduce psychological distress and fatigue in patients treated with radiotherapy ${ }^{3}$ and after high dose chemotherapy with peripheral blood stem cell transplantation. ${ }^{4}$ Furthermore, a reduction of treatment related complications has been observed in cancer patients participating in exercise programmes during cancer treatment. ${ }^{56}$ Finally, preliminary evidence suggests that regular physical activity may improve immune function. ${ }^{7-9}$ Therefore, exercise could play a potential role as complementary therapy for cancer patients during and after treatment.

However, it is necessary to have more information about the effects and feasibility of exercise programmes for different groups of patients with oncological diseases. Indeed, "cancer" is a common denominator for more than 100 neoplastic diseases, each with a different aetiology, course, and prognosis. Nevertheless, the biology of the same nosological entity may vary considerably in different settings - that is, acute lymphoblastic leukaemia in children and adults. Finally, cancer patients may have a number of specific problems. Chemotherapy can damage bone marrow and thereby impair the production of red blood cells; the resulting anaemia decreases the oxygen transport capacity of the blood. Agents like anthracyclines and cyclophosphamide, and irradiation of the mediastinum, can result in myocardial damage and therefore cause a decrease of cardiac output. Metastatic disease and pleural effusion cause a reduction of total lung capacity; furthermore, changes in the pulmonary architecture due to surgical treatment of primary or metastatic lung cancer or as a sequel to fibrosis after radiotherapy may alter the ventilation:perfusion ratio. Treatment with immunosuppressive agents (for example, high dose corticoids and cyclosporine) can lead to a marked loss of muscle mass and severe myopathy. Furthermore, reduced protein and calorie intake as a consequence of anorexia and nausea, and impaired absorption after gastrointestinal surgery, may lead to a negative nitrogen balance and hence to a catabolic state. Finally, an increase in the concentration of cytokines (IL1, IL6, TNF and IFN- $\alpha$ ) resulting from the interaction between the tumour and the host defence system has been associated with muscular waning. All these factors may affect the patient's physical condition and reduce their performance and must thus be carefully considered when designing an exercise programme.

Research about the effects of exercise in the prevention and rehabilitation of cancer and the impact of physical activity on immune function is still at its very beginning. However, we feel that this is going to be one of the most active areas of research in sports medicine in the coming decade. It is certainly time to meet the challenge. 
Freie Universitaet Berlin, Benjamin Franklin Medical Centre, Department of Sports Medicine, Clayallee 229, 14195 Berlin, Germany

1 MacVicar MG, Winningham ML, Nickel JL. Effects of aerobic interval training on cancer patients functional capacity. Nurs Res 1989;38:348-51. training on cancer patients functional capacity. Nurs Res 1989;38:348-51.
Dimeo F, Bertz H, Finke J, et al. An aerobic exercise program for patients with haematological malignancies after bone marrow transplantation. Bone Marrow Transplant 1996;18:1157-60.

3 Mock V, Dow KH, Meares CJ, et al. Effects of exercise on fatigue, physical functioning, and emotional distress during radiation therapy for breast cancer. Oncol Nurs Forum 1997;24:991-1000.

4 Dimeo F, Stieglitz R-D, Novelli-Fischer U, et al. Effects of aerobic exercise on the fatigue and psychological status of cancer patients during chemotherapy Cancer 1999:85:2273-7.

5 Winningham ML, MacVicar MG, Bondoc M, et al. Effect of aerobic exercise on body weight and composition in patients with breast cancer on adjuvant chemotherapy. Oncol Nurs Forum 1989;16:683-9.

6 Dimeo F, Fetscher S, Lange W, et al. Effects of aerobic exercise on the physical performance and incidence of treatment-related complications after high-dose chemotherapy. Blood 1997;90:3390-4.

7 Nehlsen-Cannarella SL, Nieman DC, Balk-Lamberton AJ, et al. The effects of moderate exercise training on immune response. Med Sci Sports Exer 1991;23:64-70.

8 Kaufman JC, Harris TJ, Higgins J, et al. Exercise-induced enhancement of immune function in the rat. Circulation 1994;90:525-32.

9 Crist DM, Mackinnon LT, Thompson RF, et al. Physical exercise increases natural cellular-mediated tumor cytotoxicity in elderly women. Gerontology 1989;35:66-71.

\section{The immune system in sport: getting the balance right}

The immune system consists of a vast number of cells, tissues, and messengers - for example, cytokines - that play a key role in the protection of the body against infection and in healing after injury. It is becoming increasingly evident that it is highly integrated with our neurological and endocrine systems, and research now seeks to understand and exploit these interactions. ${ }^{1}$ The need for an active immune system is self evident if an athlete is to continuously produce peak performances, but often intense exertion and treatment for inflammation lead to partially reduced immune capacity and consequently potential infection or disease. To circumvent such problems, it is of major importance to understand how to achieve the optimum balance of the immune system.

A review of the literature highlights the fact that sports immunology is now becoming a significant subdiscipline of sports science in terms of publications produced, symposia, and the development of specialist journals. Research has generated a plethora of interesting results on the effects of exercise on the immune system. It is also evident that there are variations and, in some cases, conflicts in the results published. The parameters that may modulate immune responses during exercise include nutritional status, changes in circulating levels of cytokines, the expression of adhesion molecules, changes in chemotaxis/ mobility, and the generation of reactive species. However, many of these factors are closely interlinked - for example, the role of cytokines and adhesion molecules in modulating leucocyte mobility. Immune responses are also affected by factors such as age, sex, biological rhythms, and lifestyle, and there are technical variations in the methods used to extract, purify, store, and analyse samples. There are many reports of increases/reductions in immune related cell numbers, and, although these may reflect alterations in cytokine and other levels, it is important to relate these to functional tests of activity. This is true not just in the case of sports immunology. The design of all aspects of sampling needs to ensure that results generated are highly controlled, physiologically relevant, and technically accurate. ${ }^{2}{ }^{3}$

In general, the literature suggests that acute exercisefor example, marathon and ultramarathon runningresults in an associated reduction in aspects of immune competence so that such athletes may be at increased risk of illness and need to pay particular attention to their nutritional state, hygiene, and exposure to infections. ${ }^{3}$ It has been suggested that exercise induced reductions in particular lymphocyte subsets in runners is more dependent on training intensity than volume and is transient. ${ }^{4}$ Other researchers consider that immunosuppression caused by stress as a result of acute exercise is not due to reallocation of scarce metabolic components but may represent a mechanism to reduce the potential of an autoimmune response. ${ }^{5}$ Fallon and colleagues ${ }^{6}$ concluded from their recent research that intense exercise (ultramarathon running) results in a range of alterations in haematological parameters consistent with the normal acute phase response to injury. This should not, in their view, be confused with disease, and athletes can adapt to such situations. However, for inadequately fit people, or those unwell or under medication, undertaking very strenuous exercise could be deleterious. It is also known that anaphylactic reactions may be induced by exercise in some.

There are a number of reported beneficial clinical applications of exercise to immunology in aging, cerebrovascular disease, management of acute viral infection (such as AIDS), cancer, and chronic fatigue syndrome. ${ }^{78}$ Studies in humans and experimental animals indicate that a combination of dietary restriction and physical exercise can retard age associated reductions in immunological reactivity, ${ }^{9}$ and enhancement of some immune factors was also induced by exercise in patients with cerebrovascular disease. ${ }^{10}$ Clearly the exploitation of exercise as a treatment modality is deserving of further study, and, for the athlete, the rapidly developing molecular and cellular laboratory research approaches may lead to exciting insights into how the ideal balance of the immune system may be achieved and exploited to maximise performance and health.

School of Biotechnology

RICHARD O'KENNEDY

Dublin City University

Dublin 9, Republic of Ireland

1 Sompayrac L. How the immune systems works. 1st ed. Boston: Blackwell Science, 1999.

Smith JA. Guidelines, standards and perspectives in exercise immunology. Med Sci Sports Exerc 1995;27:497-506.

3 Shephard RJ, Shek PN. Immune responses to inflammation and trauma: a physical training model. Can F Physiol Pharmacol 1998;76:469-72.

4 Kajiura JS, MacDougal JD, Ernst PB, et al. Immune-response to changes in training intensity and volume in runners. Med Sci Sports Exerc 1995;27:1111-17.

5 Raberg L, Grahn M, Hesselquist D, et al. On the adaptive significance of stress-induced immunosuppression. Proc R Soc Lond B Biol Sci 1998;265: $1637-41$.

6 Fallon KE, Sivyer G, Sivyer K, et al. Changes in haematological parameters and iron metabolism associated with a 1600 kilometre ultramarathon. $\mathrm{Br} \mathcal{F}$ Sports Med 1999;33:27-32.

7 Mackinnon LT. Future directions in exercise and immunology: Regulation and integration. Int $\mathcal{F}$ Sports Med 1998;19:5205-9.

8 Mustafe T, Sy FS, Macera CA, et al. Association between exercise and HIV disease progression in a cohort of homosexual men. Ann Epidemiol 1999;2: $127-31$.

9 Utsuyama M, Ichikawa M, Konnoshirakawa A, et al. Retardation of the ageassociated decline of immune functions in ageing rats under dietary restriction and daily physical exercise. Mech Ageing Dev 1996;91:219-28.

10 Kurabayashi H, Kubota K, Machida I, et al. Effects of physical therapy on immunological parameters in patients with cerebrovascular diseases. $\mathcal{F} \mathrm{Med}$ 1996;27:171-5. 